\title{
Práticas de enfermeiros de um hospital universitário na continuidade do cuidado para a atenção primária ${ }^{a}$
}

\author{
Practices of nurses in a university hospital for the continuity of care for primary care
}

Prácticas de las enfermeras en un hospital universitario en la continuidad de la atención primaria

\author{
Lays Souza de Oliveira ${ }^{1}$ (1) \\ Maria Fernanda Baeta Neves \\ Alonso da Costa 2,3 (C) \\ Patrícia Madalena Vieira Hermida 4 (1) \\ Selma Regina de Andrade $^{3}$ (B) \\ Juanah Oliveira Debetio ${ }^{3}$ (D) \\ Larissa Martins Novaes de Lima $^{3}$ [D
}

1. Universidade Federal de Santa Catarina.

Florianópolis, SC, Brasil.

2. Universidade Federal de Santa Catarina,

Departamento de Enfermagem. Florianópolis, SC, Brasil.

3. Universidade Federal de Santa Catarina,

Pós-graduação em Enfermagem. Florianópolis, SC, Brasil.

4. Secretaria Municipal de Saúde da Prefeitura de Florianópolis. Florianópolis, SC, Brasil.

Autor correspondente:

Maria Fernanda Baeta Neves Alonso da Costa.

E-mail: fernanda.baeta@ufsc.br

Recebido em 28/12/2020.

Aprovado em 05/06/2021.

DOI:https://doi.org/10.1590/2177-9465-EAN-2020-0530

\begin{abstract}
Resumo
Objetivo: compreender as práticas dos enfermeiros de um hospital universitário na continuidade do cuidado para a atenção primária. Método: estudo exploratório, descritivo e qualitativo, realizado entre agosto e novembro de 2019, em hospital universitário no Sul do Brasil, com 21 enfermeiros e a diretora de Enfermagem, aplicando-se, respectivamente, um instrumento on-line na plataforma Survey Monkey e uma entrevista semiestruturada. Os dados coletados foram submetidos à Análise de Conteúdo segundo Minayo. Resultados: emergiram três categorias: práticas dos enfermeiros; fortalezas e fragilidades e competências para a continuidade do cuidado. Na admissão e alta, os enfermeiros realizam entrevista e exame físico do paciente. Os pontos positivos foram a comunicação e o conhecimento do contexto familiar da equipe multiprofissional hospitalar e os negativos, a falta de sistema informatizado, a integração dos profissionais do hospital com a atenção primária, o enfermeiro gestor de altas e o protocolo de contrarreferência. A continuidade do cuidado requer, dos enfermeiros, experiência profissional, conhecimento sobre a rede de atenção, habilidades de comunicação, liderança e tomada de decisão. Conclusão e implicações para a prática: os enfermeiros compreendem a importância da continuidade do cuidado, entretanto, algumas fragilidades encontradas na instituição dificultam a realização dessas práticas.
\end{abstract}

Palavras-chave: Continuidade da Assistência ao Paciente; Cuidado Transicional; Alta do Paciente; Hospitais; Atenção Primária à Saúde.

\section{Abstract}

Objective: to understand the practices of nurses from a university hospital in the continuity of care for primary care. Method: an exploratory, descriptive, and qualitative study, conducted between August and November 2019, in a university hospital in Southern Brazil, with 21 nurses and the director of Nursing, applying, respectively, an online instrument in the Survey Monkey platform and a semi-structured interview. The data collected was submitted to Content Analysis according to Minayo. Results: three categories emerged: nurses' practices; strengths and weaknesses, and competencies for continuity of care. On admission and discharge, nurses interview and physically examine the patient. The positive aspects were communication and knowledge of the family context by the multiprofessional hospital team, and the negative aspects were the lack of a computerized system, the integration of hospital professionals with primary care, the nurse discharge manager and the counter-reference protocol. Continuity of care requires from nurses professional experience, knowledge about the care network, communication skills, leadership, and decision making. Conclusion and implications for practice: nurses understand the importance of continuity of care, however, some weaknesses found in the institution make it difficult to carry out these practices.

Keywords: Continuity of Patient Care; Transitional Care; Patient Discharge; Hospitals; Primary Health Care.

\section{REsumen}

Objetivo: conocer las prácticas de los enfermeros de un hospital universitario en la continuidad de los cuidados de la atención primaria. Método: estudio exploratorio, descriptivo y cualitativo, realizado entre agosto y noviembre de 2019, en un hospita universitario del sur de Brasil, con 21 enfermeros y la Directora de Enfermería, aplicando, respectivamente, instrumento online en la plataforma Survey Monkey y una entrevista semiestructurada. Los datos recogidos se sometieron a un análisis de contenido según Minayo. Resultados: emergiron tres categorías: prácticas de los enfermeros; fortalezas y fragilidades y competencias para la continuidad del cuidado. En el momento del ingreso y del alta, los enfermeros entrevistan y realizan una exploración física del paciente. Los aspectos positivos fueron la comunicación y el conocimiento del contexto familiar por parte del equipo multiprofesional del hospital, y los negativos la falta de un sistema informatizado; la integración de los profesionales del hospital con la atención primaria; la enfermera gestora del alta y el protocolo de contrarreferencia. La continuidad de los cuidados requiere la experiencia profesional de los enfermeros, el conocimiento de la red asistencial, la capacidad de comunicación, el liderazgo y la toma de decisiones. Conclusión e implicaciones para la práctica: Los enfermeros entienden la importancia de la continuidad de los cuidados, sin embargo, algunas debilidades encontradas en la institución dificultan la implementación de estas prácticas

Palabras clave: Continuidad asistencial; Atención transitoria; Alta de pacientes; Hospitales; Atención primaria. 


\section{INTRODUÇÃO}

A continuidade do cuidado pode ser conceituada como o maior nível de conexão entre os serviços do sistema de saúde experimentado pelos pacientes ${ }^{1}$. Nesse sentido, no Brasil, foi criada a Rede de Atenção à Saúde (RAS) na tentativa de integrar os diferentes serviços de saúde, de forma horizontal, com todos os pontos da rede, com um cuidado contínuo, integral e de qualidade².

A melhoria da qualidade dos serviços contribui para a redução dos custos e de hospitalizações evitáveis, a superação da fragmentação da assistência e a continuidade do cuidado ${ }^{3-4}$, a qual depende de fatores como: boa comunicação e troca de informações entre profissionais e pacientes; interdisciplinaridade; articulação entre os pontos da RAS; coordenação do cuidado e corresponsabilização do paciente acerca da sua saúde ${ }^{5}$.

A continuidade do cuidado dirige-se aos pacientes, familiares e cuidadores e aos profissionais da saúde que prestam a assistência. Trata-se de um processo que requer, sobretudo, coordenação e comunicação entre diferentes profissionais e pessoas, considerandose suas experiências e habilidades ${ }^{4}$, aspectos relevantes para a transição do cuidado de um ponto da rede de cuidado para outro, especialmente em tempos de pandemia, quando a segurança, o distanciamento social e a realização dos cuidados necessários devem ser priorizados com cautela ${ }^{6}$.

Quando a continuidade do cuidado ocorre do hospital para a Atenção Primária à Saúde (APS), há a necessidade de realizar o planejamento da alta pela equipe multidisciplinar, bem como as orientações para os cuidados no domicílio ${ }^{7}$. No plano de alta, os enfermeiros orientam o paciente e a família para a promoção do autocuidado ${ }^{8}$. Entretanto, enfermeiros brasileiros relatam dificuldades para realizar a continuidade do cuidado devido ao desconhecimento da RAS, à ausência de protocolos clínicos e de contrarreferência ${ }^{8-9}$.

Na Espanha, Portugal e Canadá, os enfermeiros realizam a articulação do serviço hospitalar com a APS e são preparados para promover a continuidade do cuidado ${ }^{10}$. Nesses países, as Enfermeiras Hospitalares de Enlace (EHE) e as Enfermeiras de Ligação realizam a avaliação clínica e social do paciente e utilizam protocolos na alta hospitalar ${ }^{11}$.

Na Espanha, a EHE realiza esta articulação com a enfermeira da APS por meio de plataforma eletrônica hospitalar, tablet ou pelo telefone ${ }^{11}$. Em Portugal e no Canadá, a Enfermeira de Ligação identifica os recursos necessários na pós-alta para o cuidado no domicílio e realiza o contato com os profissionais da APS. Esta enfermeira conta com um sistema informático que compartilha as informações do paciente com um serviço extra-hospitalar que, posteriormente, realiza os devidos encaminhamentos. Assim, a transferência das informações sobre o paciente é realizada pela Enfermeira de Ligação, majoritariamente, por meio do envio eletrônico do formulário de contrarreferência a um serviço extra-hospitalar ${ }^{12}$.

No Brasil, em Curitiba, foi implementada a Enfermeira de Ligação que, desde a internação, avalia os pacientes que necessitam da continuidade do cuidado para a $\mathrm{APS}^{9-10}$. Contudo, a fragmentação na transição/continuidade do cuidado ainda merece discussões ${ }^{13}$. Observa-se que, na maioria das instituições hospitalares brasileiras, não existe enfermeiro que desempenha especificamente o papel de Enfermeiro de Ligação ou transição.

Objetivou-se, por este estudo, considerando que a continuidade do cuidado deve ocorrer de acordo com as necessidades dos indivíduos e a organização dos serviços, compreender: "Como ocorre, em um hospital universitário, as práticas dos enfermeiros na continuidade do cuidado para a Atenção Primária à Saúde?".

\section{MÉTODO}

Estudo exploratório e descritivo, de abordagem qualitativa, realizado em um hospital universitário do Sul do Brasil, caracterizado como um hospital geral de média e alta complexidade, com 238 leitos ativos e que oferece tratamentos clínicos e cirúrgicos, de Ginecologia e Obstetrícia, com alojamento conjunto, berçário, Pediatria, Unidade de Terapia Intensiva (UTI) adulto e neonatal e emergências adulto, obstétrica/ginecológica e pediátrica.

Dentre os profissionais de Enfermagem, 263 são enfermeiros, com distintas funções ligadas à assistência, gestão e educação. $\mathrm{Na}$ assistência, o enfermeiro realiza a alta hospitalar, embora inexista o cargo de enfermeiro gestor de alta nessa instituição.

Participaram da pesquisa a diretora de Enfermagem e 21 enfermeiros, dentre os 45 convidados por conveniência, atendendo aos critérios de inclusão e exclusão. Foram incluídos os enfermeiros que trabalhavam nas unidades de Clínica Cirúrgica, Clínica Médica, Clínica Pediátrica, Alojamento Conjunto, Ginecologia, Emergência adulto e pediátrica, que não se encontravam de férias ou licença. Foram excluídos aqueles que trabalhavam no turno da noite, devido ao número reduzido de altas hospitalares nesse período, e os enfermeiros das Unidades de Terapia Intensiva adulto e neonatal, Centro Cirúrgico e Obstétrico e Emergência Obstétrica, em razão das altas destas serem intra-hospitalares. A Unidade de Hemodiálise e os ambulatórios foram excluídos por não haver internação nestes setores.

Os enfermeiros foram convidados pessoalmente para participar do estudo, momento em que se apresentou o projeto e foi solicitado o e-mail para, posteriormente, o encaminhamento do Termo de Consentimento Livre e Esclarecido (TCLE) e do formulário de coleta de dados na plataforma Survey Monkey, ferramenta eletrônica de acesso privado que tem como proposta a criação, aplicação, coleta e análise dos dados via internet com privacidade e segurança dos dados ${ }^{14}$.

Este estudo integra um estudo multicêntrico "As práticas da Enfermeira de Enlace para a continuidade do cuidado: estudo multicêntrico", com a aplicação de um mesmo formulário de coleta junto aos enfermeiros assistenciais da instituição em tela. O formulário possui 59 questões abertas e fechadas. Os principais temas abordados foram: perfil dos participantes, comunicação entre os enfermeiros hospitalares e as competências como enfermeiro de continuidade do cuidado.

O formulário ficou disponível entre agosto e novembro de 2019 e, a cada sete dias, os enfermeiros, que ainda não haviam respondido, receberam uma mensagem de lembrete na plataforma enviada pelas pesquisadoras. 
Para a coleta de dados com a diretora de Enfermagem, foi realizada a entrevista pessoalmente, utilizando-se, como guia, um roteiro com perguntas abertas acerca do perfil da participante e sobre a gestão de alta e continuidade do cuidado. A entrevista foi gravada em dispositivo de áudio e transcrita na íntegra para um documento no formato Microsoft Word ${ }^{\circledR}$.

Os dados foram analisados com base na Análise de Conteúdo de Minayo ${ }^{15}$ e, a partir da junção de elementos principais, formaram-se categorias, as quais se relacionam com os objetivos da pesquisa e a revisão de literatura sobre o tema. Para proceder à análise, extraíram-se as respostas dos participantes da Plataforma Survey Monkey e realizou-se a transferência para uma planilha no formato de Microsoft Exce ${ }^{\circledR}$.

O estudo atendeu à Resolução $n^{\circ}$ 466, de 12 de dezembro de 2012, e é um recorte da pesquisa "As práticas da Enfermeira de Enlace para a continuidade do cuidado: estudo multicêntrico", aprovada pelo Comitê de Ética em Pesquisa com Seres Humanos, da Universidade Federal de Santa Catarina (UFSC), sob o Parecer $\mathrm{n}$ - 3.413.257. Para garantir o anonimato dos profissionais, nomes de praias foram utilizados para nomear os participantes.

\section{RESULTADOS}

Dos 21 participantes, 19 eram do sexo feminino, com idade média de 36 anos e tempo de atuação como enfermeiros entre oito meses e 21 anos, sendo que dez possuem especialização; oito possuem mestrado; um, doutorado. Sobre o tempo de experiência como "Enfermeiro Gestor de Altas", oito responderam não ter experiência, oito, o mesmo tempo de atuação como enfermeiro, e cinco, entre cinco meses e oito anos.

Da análise dos dados, emergiram três categorias: práticas desenvolvidas pelos enfermeiros hospitalares para a continuidade do cuidado; fortalezas e fragilidades para a continuidade do cuidado e competências desenvolvidas para a continuidade do cuidado.

\section{Práticas desenvolvidas pelos enfermeiros hospitalares para a continuidade do cuidado}

No hospital do estudo, não existe o cargo de enfermeiro gestor de altas. Os enfermeiros assistenciais relataram que nem sempre realizam o planejamento da alta devido à sobrecarga de trabalho e à ausência de protocolos clínicos na instituição, mas reconhecem que é sua atribuição.

\section{[...] nós não temos essa enfermeira com essa atividade específica [...]. (Gravatá) \\ [...] As enfermeiras assistenciais realizam a gestão da alta [...]. (Mole) \\ [...] Excesso de trabalho, emergência lotada, resolução de assuntos administrativos, reduzindo período da assistência [...]. (Joaquina)}

A maioria dos participantes expressou a necessidade de cuidados continuados nas visitas diárias aos pacientes, coleta dos dados clínicos no prontuário e informações da família. Todas as enfermeiras realizam o exame físico e a entrevista com os pacientes e familiares na qual consideram a história pregressa, as comorbidades, a situação socioeconômica, a rede de apoio e a avaliação da capacidade funcional para realizar as atividades de vida diária.

[...] Visita e avaliação diária e observação das necessidades de cada paciente [...]. (Ponta das Canas)

[...] A partir da história de vida do paciente, das suas condições socioeconômicas e clínicas, tentamos prever possíveis medidas de proteção para continuidade do cuidado e evitar reinternações. [...]. (Naufragados)

[...] dados pessoais, motivo pelo qual procurou atendimento, comorbidades, antecedentes pessoais, hábitos, exame físico sucinto, necessidades de prevenção de quedas ou de fuga e isolamento etc. [...]. (Barra da Lagoa)

[...] Percepções e expectativas, problemas relacionados às necessidades humanas básicas [...]. (Matadeiro)

Apenas seis enfermeiras consideraram o planejamento da alta função do médico e do assistente social. Algumas enfermeiras relataram que o início do planejamento da alta ocorre desde a admissão do paciente no hospital. Já outras realizam durante a internação ou somente no momento da alta hospitalar, em conjunto com a equipe multidisciplinar. Os principais profissionais envolvidos na alta hospitalar são os médicos seguidos dos assistentes sociais e enfermeiros. No planejamento da alta hospitalar, a maioria dos enfermeiros entrevista os familiares para compreender as condições em que seriam realizados os cuidados no domicílio.

[...] Em planos que envolvem mudanças intensas no contexto familiar [...], é realizado uma conferência para discussão das necessidades. Geralmente, nesse momento, estão assistente social, psicólogo, enfermeiro, médico e familiares[...]. (Santinho)

[...] Respondendo questionamentos de como este doente ficará em casa, com quem, aos cuidados de quem, se tem condição financeira para mantê-lo, se é possível retornar para o ambulatório, onde mora etc. [...]. (Açores)

$\mathrm{Na}$ alta hospitalar, alguns cuidados foram prescritos pelo médico e enfermeiros, entre eles: curativos; cuidados com sondas e colostomia; cuidados com a pele; receituário médico com a prescrição dos medicamentos; pedidos de exames e encaminhamento para consultas com especialistas.

[...] Formulário de curativo: explica como foi realizado o último curativo no hospital, sugere a cobertura a ser utilizada e descreve como deve ser realizado o curativo, período de troca [...]. (Mole)

[...] Sumário de alta hospitalar, cartão de alta, receituário médico, encaminhamento para consultas, exames etc. [...]. (Brava) 
Raramente, há comunicação entre os profissionais do hospital e da APS e, quando ocorre, dá-se por meio do serviço social do hospital. As informações do paciente são fornecidas pelos médicos na forma de resumo de alta impresso, documento que requer, do paciente, a responsabilidade por levar as informações para os profissionais da APS.

[...] Só em casos muito específicos é que se faz este contato com a Unidade Básica. Mas, geralmente, não se faz [...]. (Gravatá)

[...] O paciente portando a caderneta de saúde ou impressos fornecidos pelo hospital [...]. (Brava)

[...] Acredito que nenhuma por escrito, pois não há referência e contrarreferência. Porém, quando necessário, e se há tempo, é entrado em contato via telefone com a UBS [...]. (Santinho)

Todos os enfermeiros afirmaram não conhecer indicadores que avaliam, de maneira adequada, a continuidade do cuidado do paciente para a APS, entretanto, a maioria revelou monitorar o paciente pós-alta mediante o contato telefônico e o agendamento de consultas e exames no ambulatório do hospital.

Quando ocorre a reinternação, o profissional de saúde só tem conhecimento do fato quando o paciente é novamente admitido na mesma clínica. Na reinternação, a maioria das enfermeiras afirmou que não informa o profissional da APS, mesmo que possua conhecimento prévio sobre os recursos necessários à continuidade do cuidado no domicílio, uma vez que elas já trabalharam em outro serviço de saúde ou na APS.

[...] Por conversas informais com o Serviço Social, Medicina, colegas enfermeiros da rede básica de saúde [...]. (Santinho)

[...] Por já ter trabalhado na atenção primária [...]. (Solidão)

[...] Informações de colegas e internet [...]. (Lagoinha do Leste)

[...] Sabemos da reintegração somente quando reinterna na mesma clínica [...]. (Moçambique)

\section{Fortalezas e fragilidades para a continuidade do cuidado}

Dentre as fortalezas encontradas pelas enfermeiras para a continuidade do cuidado estão a boa comunicação da equipe multiprofissional e o conhecimento sobre o contexto familiar no qual o paciente está inserido.

[...] Boa comunicação entre a equipe multiprofissional [...]. (Mole)

[...] Existência de equipe multiprofissional dentro do hospital [...]. (Brava)
[...] Conhecimento do contexto familiar do paciente [...]. (Santinho)

As fragilidades mais citadas pelas enfermeiras foram o excesso de trabalho e de processos administrativos, a falta de integração dos profissionais do hospital com os da APS, a ausência de enfermeiro que realize a gestão da alta hospitalar, a inexistência de indicadores e de protocolos de alta hospitalar e de contrarreferência, assim como a ausência de prontuário eletrônico e sistema informatizado integrado entre o hospital e a APS.

[...] Falta de comunicação, interação com outros profissionais, sobrecarga de atividades [...]. (Brava)

[...] Falta de tempo. (Mole)

[...] o prontuário único iria ajudar muito, iria ajudar demais. Então, se fosse elencar agora a maior dificuldade, eu diria que é esse prontuário, a falta desse prontuário único [...]. (Gravatá)

[...] Falta de um profissional enfermeiro específico para tal função[...]. (Matadeiro)

\section{Competências para a continuidade do cuidado}

Os enfermeiros expressaram que a competência para a continuidade do cuidado requer prática assistencial, plano de cuidado individualizado e centrado no paciente, conhecimento técnico-científico sobre RAS e os protocolos implementados na instituição hospitalar, atenção integral, comunicação clara e efetiva com o paciente, família e profissionais, participação em reuniões de equipe, espírito de liderança e tomador de decisões.

Para desenvolver essas competências, são essenciais treinamentos ou capacitações com os enfermeiros acerca da gestão do cuidado, comunicação e informática. No hospital em estudo, essas capacitações podem constituir as ações de educação permanente em saúde implementadas pelo Serviço de Educação Continuada.

[...] Capacitações, treinamentos, conhecimento em várias fontes[...]. (Barra da Lagoa)

[...] Com uma comunicação ativa entre todos os envolvidos no cuidado do paciente [...]. (Açores)

\section{DISCUSSÃO}

O perfil dos enfermeiros deste estudo assemelha-se ao identificado em Portugal sobre a Enfermeira de Ligação em que a maioria possui idades entre 35 e 44 e é do gênero feminino.

A literatura destaca a relevância de se ter, no hospital, um profissional que desempenhe a função de coordenar as altas e articular a continuidade do cuidado com os demais pontos da RAS, em especial, com a APS ${ }^{12}$.

Apesar disso, os enfermeiros assistenciais conseguem avaliar os pacientes que necessitam de cuidados continuados 
após a alta hospitalar, avaliação que é realizada durante as visitas diárias, na busca dos diagnósticos no prontuário e na comunicação com paciente e familiares. Nas instituições hospitalares espanholas, as EHE são responsáveis por identificar, nos pacientes internados, aqueles que requerem continuidade do cuidado na APS, levantar os recursos necessários para o cuidado no domicílio e orientar para o autocuidado ${ }^{16}$. As Enfermeiras de Ligação de Portugal avaliam os pacientes que necessitam de cuidados mais complexos no momento da visita diária quando fazem o levantamento dos dados clínicos e realizam o exame físico. Recomenda-se que a avaliação do paciente ocorra na sua admissão ou até nas primeiras 48 horas da internação para que se inicie o planejamento da alta hospitalar ${ }^{17}$.

A avaliação inicial e contínua das necessidades do paciente permite, ao enfermeiro, planejar, de maneira adequada, a continuidade do cuidado, além de promover vínculo com o paciente e sua família $^{18}$. Sobre o planejamento da alta, possibilita ofertar atividades e informações individualizadas centradas no paciente. Contudo, a ausência de um protocolo de plano de alta ou contrarreferência dificulta a continuidade do cuidado. Em hospitais onde há um protocolo de plano de alta individualizado desde a internação, houve a redução das reinternações hospitalares, as quais ocorrem devido à má comunicação entre os profissionais, o paciente e a família sobre as orientações e os cuidados no domicílio ${ }^{19}$.

Nesse meandro, ressalta-se que as ações de educação em saúde, realizadas pela enfermeira durante o planejamento da alta são essenciais para promover o autocuidado e o empoderamento do paciente e de seus familiares, compartilhar conhecimentos, sanar dúvidas relacionadas ao processo saúde-doença e realizar as orientações pertinentes aos cuidados no domicílio ${ }^{20}$.

Embora alguns enfermeiros realizem o planejamento de alta, não ficou claro, nesta pesquisa, que o preparo do paciente para a alta ocorra por meio da educação em saúde. Entretanto, os enfermeiros têm uma boa comunicação com os pacientes e familiares de modo que conhecem o contexto familiar e a rede de apoio. Ainda sobre o preparo para a alta, um estudo destacou a relevância dos enfermeiros envolverem os familiares no cuidado durante a internação com vistas aos cuidados adequados no domicílio ${ }^{21}$. O envolvimento da família nas intervenções recomendadas para o pós-alta hospitalar garante o sucesso do plano de cuidados e da continuidade do cuidado no domicílio ${ }^{19}$.

No momento da alta hospitalar, os pacientes recebem um resumo impresso da internação elaborado pelos médicos e raramente há comunicação da equipe de saúde do hospital com os profissionais da APS e, quando ocorre, esta é realizada pelo assistente social. O compartilhamento de informações entre os serviços é essencial para promover a continuidade do cuidado e garantir que os profissionais da APS tenham conhecimento dos pacientes submetidos à internação hospitalar. Essa comunicação pode ser realizada por meio de e-mails, telefonemas, relatórios de alta detalhado e sistema informatizado integrado ${ }^{21}$. No pós-alta, o acompanhamento por contato telefônico, visitas domiciliares ou serviços de assistência domiciliar são importantes para identificar e prover recursos mais adequados ${ }^{18}$ à continuidade do cuidado.
A qualidade do cuidado depende de fatores como competência técnica e habilidade de interação e comunicação dos profissionais com os pacientes, familiares e com outros profissionais. Dentre as fortalezas reveladas pelos participantes para a continuidade do cuidado está a comunicação entre os profissionais da equipe multiprofissional. Um estudo realizado na Espanha com EHE corroborou a comunicação como um elemento facilitador do processo de continuidade do cuidado, assim como a interação entre os profissionais e o trabalho em equipe ${ }^{22}$

O conhecimento sobre o contexto familiar do paciente também emergiu nesta investigação como facilitador para a continuidade do cuidado. Um estudo destacou que entender a rede de apoio do paciente é fundamental para compreender como decorrerá o cuidado pós-alta hospitalar, além de possibilitar uma educação em saúde individualizada para o autocuidado dos pacientes e família ${ }^{8}$

Para os enfermeiros deste estudo, a falta de tempo e a sobrecarga de trabalho são algumas das fragilidades para a continuidade do cuidado, as quais, em geral, estão relacionadas com a falta de recursos humanos ${ }^{23}$. O quantitativo limitado de profissionais, a extensa carga horária e a sobrecarga de trabalho colaboram e influenciam diretamente a qualidade do cuidado oferecido pelos enfermeiros. Ainda, a sobrecarga de trabalho interfere na produção de ações criativas e efetivas, com prejuízos à garantia do acesso integral nos pontos de atenção ${ }^{24}$ e à continuidade do cuidado.

Outra fragilidade evidenciada pelos participantes deste estudo refere-se à falta de articulação entre os profissionais, da referência e contrarreferência entre os diferentes pontos da RAS e do acompanhamento do paciente na APS pós-alta hospitalar, aspectos que fortalecem a fragmentação do cuidado ${ }^{20}$. Essa fragilidade, da mesma forma, foi encontrada na literatura com relação ao cuidado com os neonatos ${ }^{25}$.

O serviço de contrarreferência de um hospital quaternário do Sul de Minas Gerais, implementado em 2017, sinalizou o desafio de manter um sistema integrado e efetivo. Para melhorar essa realidade, julgam-se, como necessárias, a reestruturação dos serviços de referência e contrarreferência, a educação permanente sobre a temática e a criação de um prontuário único entre todos os níveis de atenção e em todo o Brasili ${ }^{26}$.

Uma pesquisa corroborou o prontuário eletrônico unificado como ferramenta que facilita a comunicação entre pontos distintos da RAS ${ }^{27}$. Protocolos de contrarreferência também podem facilitar esse diálogo entre os serviços de densidades tecnológicas diferentes. Nesse sentido, as Enfermeiras de Ligação, que trabalham no Hospital Universitário em Curitiba, implementaram um protocolo de contrarreferência em conjunto com a Secretaria Municipal de Saúde. Na alta hospitalar, realizam o contato telefônico com a enfermeira da APS para discutir as necessidades do indivíduo e agendar uma consulta. Em seguida, é preenchido o formulário de contrarreferência em duas vias, anexado ao resumo da alta e folha de rosto, em que uma via é encaminhada para a coordenação da APS, via e-mail, e outra entregue para o paciente. A implementação do protocolo de contrarreferência foi considerada muito positiva porque possibilita a integração entre os pontos da RAS e a continuidade do cuidado ${ }^{\text {. }}$ 
Este estudo revelou as competências que devem ser desenvolvidas nos enfermeiros para atuarem na continuidade do cuidado: comunicação efetiva com paciente, familiar e com outros profissionais; educação em saúde; trabalho em equipe multidisciplinar; conhecimento técnico e científico e habilidades de liderança, supervisão e capacidade de articulação com a rede. A despeito disso, a literatura complementa que há a necessidade de capacitação do enfermeiro para coordenar os casos mais complexos que requerem a continuidade do cuidado ${ }^{16,28}$. A qualificação dos profissionais é essencial para promover uma assistência de qualidade, atingir o objetivo da gestão do cuidado e proporcionar um cuidado integral ${ }^{29}$.

Em consonância com os achados, a liderança é uma das principais competências que o enfermeiro precisa aprimorar, pois, em geral, é ele quem desenvolve o papel de líder da sua equipe. $\mathrm{O}$ ato de liderar está presente em todas as atividades do enfermeiro, da organização do serviço e da relação com outros profissionais à tomada de decisão. No ambiente hospitalar, a habilidade de liderar causa impacto no serviço pelo fato de os enfermeiros comumente ocuparem cargos de chefia ${ }^{29-30}$.

A comunicação também é uma competência importante para o enfermeiro, pois o trabalho em equipe exige, dos enfermeiros, a troca de informações com a equipe multiprofissional, o paciente $e$ o familiar ${ }^{29}$, além de influenciar diretamente na articulação com os outros pontos da rede. Atrelada a isso, a educação em saúde que o enfermeiro realiza com o paciente e os familiares viabiliza-se com o compartilhamento de informações de forma clara e efetiva, utilizando diferentes recursos de comunicação e garantindo que todas as dúvidas foram sanadas para, dessa forma, diminuir os níveis de ansiedade acerca do autocuidado após a alta hospitalar ${ }^{31}$

O enfermeiro desempenha papel relevante na continuidade dos cuidados entre os serviços de saúde de diferentes densidades tecnológicas. Desse modo, deve-se ampliar, valorizar e qualificar o serviço da Enfermagem, visando a transições do cuidado cada vez mais eficientes ${ }^{4}$.

A pesquisa limitou-se ao cenário de um hospital de ensino e, sobretudo, aos enfermeiros assistenciais, aspecto que se deve ao fato de não haver enfermeiro gestor de alta na instituição investigada. Todavia, o estudo vislumbra o importante papel do enfermeiro hospitalar na continuidade do cuidado para a APS e os desafios da contrarreferência nesse processo de transição do cuidado.

\section{CONCLUSÃO E IMPLICAÇÕES PARA A PRÁTICA}

As práticas desenvolvidas pelos enfermeiros de um hospital de ensino para a continuidade do cuidado são: visitas diárias aos pacientes internados, entrevista e exame físico, além do planejamento da alta hospitalar desde a internação. No momento da alta, os pacientes recebem o resumo impresso da mesma com informações importantes da internação e dos cuidados necessários no domicílio. Raramente, se realiza contato por e-mail ou telefone com os profissionais da APS para discussão e/ou acompanhamento dos pacientes por esse ponto da RAS.
A boa comunicação entre a equipe multiprofissional do hospital e o conhecimento sobre o contexto familiar dos pacientes foram evidenciados como fortalezas para a continuidade do cuidado. Por outro lado, a falta de integração entre os profissionais do hospital e da APS, a inexistência de um prontuário eletrônico integrado, de enfermeiro de gestão da alta, de protocolos para a realização desta e de contrarreferência estão entre as fragilidades para a continuidade do cuidado.

A continuidade do cuidado requer dos enfermeiros: prática profissional; conhecimento técnico-científico sobre a RAS e os protocolos institucionais; habilidades de comunicação, de liderança e de tomada de decisão. Estas competências podem ser desenvolvidas por meio de capacitações e oportunizadas pelo Serviço de Educação Permanente do hospital.

Esta pesquisa possibilitou compreender que a continuidade do cuidado vem sendo cada vez mais abordada na literatura, entretanto, na prática, ainda existem muitos obstáculos que interferem na continuidade do cuidado do hospital para a atenção primária.

Os objetivos desta pesquisa foram alcançados, uma vez que foi possível compreender as práticas dos enfermeiros de um hospital universitário na continuidade do cuidado para a APS.

Os resultados apontaram algumas fragilidades, dentre elas, a falta de protocolo de plano de alta e do enfermeiro coordenador do cuidado, além da ausência de protocolo de contrarreferência e de um prontuário eletrônico unificado com acesso em toda a RAS.

Percebeu-se que os profissionais sabem da importância de realizar a continuidade do cuidado para a APS, principalmente dos pacientes complexos e que necessitam de cuidados domiciliares.

Concluiu-se que novas pesquisas são necessárias para que se concretize a continuidade do cuidado no contexto hospitalar e na APS com o objetivo de superar a fragmentação da RAS e garantir a integralidade do cuidado.

\section{CONTRIBUIÇÕES DOS AUTORES}

Desenho do estudo. Lays Souza de Oliveira. Maria Fernanda Baeta Neves Alonso da Costa.

Coleta ou produção dos dados. Lays Souza de Oliveira.

Análise de dados. Lays Souza de Oliveira. Maria Fernanda Baeta Neves Alonso da Costa. Patrícia Madalena Vieira Hermida. Selma Regina de Andrade. Juanah Oliveira Debeti. Larissa Martins Novaes de Lima.

Interpretação dos resultados. Patrícia Madalena Vieira Hermida. Selma Regina de Andrade. Juanah Oliveira Debeti. Larissa Martins Novaes de Lima.

Redação e revisão crítica do manuscrito. Patrícia Madalena Vieira Hermida. Selma Regina de Andrade. Juanah Oliveira Debeti. Larissa Martins Novaes de Lima.

Aprovação da versão final do artigo. Patrícia Madalena Vieira Hermida. Selma Regina de Andrade. Juanah Oliveira Debeti. Larissa Martins Novaes de Lima.

Responsabilidade por todos os aspectos do conteúdo e pela integridade do artigo publicado. Lays Souza de Oliveira. Maria Fernanda Baeta Neves Alonso da Costa. Patrícia Madalena Vieira 
Hermida. Selma Regina de Andrade. Juanah Oliveira Debeti. Larissa Martins Novaes de Lima.

\section{EDITOR ASSOCIADO}

\author{
Gerson Luiz Marinho (D)
}

\section{EDITOR CIENTÍFICO}

\author{
Ivone Evangelista Cabral (B)
}

\section{REFERÊNCIAS}

1. Vargas I, Mogollón-Pérez AS, Paepe P, Silva MRF, Unger J, Vázquez ML Barriers to healthcare coordination in market-based and decentralized public health systems: a qualitative study in healthcare networks of Colombia and Brazil. Health Policy Plan. 2016 fev;31(6):736-48. http:// dx.doi.org/10.1093/heapol/czv126. PMid:26874327.

2. Peiter CC, Santos JLG, Lanzoni GMM, Mello ALSF, Costa MFBNA, Andrade SR. Healthcare networks: trends of knowledge development in Brazil. Esc Anna Nery. 2019;23(1):e20180214. http://dx.doi. org/10.1590/2177-9465-ean-2018-0214.

3. Mendes EV. Entrevista: a abordagem das condições crônicas pelo Sistema Único de Saúde. Cien Saude Colet.2018 fev;23(2):431-6. http:// dx.doi.org/10.1590/1413-81232018232.16152017. PMid:29412401.

4. Weber LAF, Lima MADS, Acosta AM, Marques GQ. Transição do cuidado do hospital para o domicílio: revisão integrativa. Cogitare Enferm. 2017 jul;22(3):e47615. http://dx.doi.org/10.5380/ce.v22i3.47615.

5. Utzumi FC, Lacerda MR, Bernardino E, Gomes IM, Aued GK, Sousa SM. Continuidade do cuidado e o interacionismo simbólico: um entendimento possível. Texto Contexto Enferm. 2018 mai;27(2):e4250016. http:// dx.doi.org/10.1590/0104-070720180004250016.

6. Knihs NS, Sens S, Silva AM, Wachholz LF, Paim SMS, Magalhães ALP. Transição do cuidado de pacientes submetidos ao transplante hepático durante a pandemia da COVID-19. Texto Contexto Enferm. 2020;29:e20200191. http://dx.doi.org/10.1590/1980-265x-tce-2020-0191.

7. Meyers AG, Salanitro A, Wallston KA, Cawthon C, Vasilevskis EE, Goggins $\mathrm{KM}$ et al. Determinants of health after hospital discharge: rationale and design of the Vanderbilt Inpatient Cohort Study (VICS). BMC Health Serv Res. 2014 jan;14(1):10. http://dx.doi.org/10.1186/1472-6963-1410. PMid:24397292.

8. Acosta AM, Câmara CE, Weber LAF, Fontenele RMF. Atividades do enfermeiro na transição do cuidado: realidades e desafios. Rev Enferm UFPE. 2018 dez;12(12):3190-6. http://dx.doi.org/10.5205/1981-8963v12i12a231432p3190-3197-2018.

9. Ribas EN, Bernardino E, Larocca LM, Poli No P, Aued GK, Silva CPC. Nurse liaison: a strategy for counter-referral. Rev Bras Enferm. 2018;71(suppl 1):546-53. http://dx.doi.org/10.1590/0034-7167-20170490. PMid:29562010.

10. Amendola F. Integralidade da assistência ao paciente cirúrgico: articulação entre o hospital e a atenção primária. Rev SOBECC. 2019 dez;24(4):173-4. http://dx.doi.org/10.5327/Z1414-4425201900040001.

11. Costa MFBNA, Andrade SR, Soares CF, Pérez EIB, Tomás SC, Bernardino E. The continuity of hospital nursing care for Primary Health Care in Spain. Rev Esc Enferm USP. 2019;53:e03477. http://dx.doi. org/10.1590/s1980-220x2018017803477. PMid:31291396.

12. Aued GK, Bernardino E, Lapierre J, Dallaire C. Atividades das enfermeiras de ligação na alta hospitalar: uma estratégia para a continuidade do cuidado. Rev Latinoam Enferm. 2019;27:e3162. http://dx.doi. org/10.1590/1518-8345.3069.3162.

13. Hadad ACAC, Jorge AO. Continuidade do cuidado em rede e os movimentos de redes vivas nas trajetórias do usuário-guia. Saude Debate. 2018 dez;42(spe4):198-210. http://dx.doi.org/10.1590/0103-11042018s416.
14. Surveymonkey. SurveyMonkey: a ferramenta de questionários online mais popular do mundo. 2020 [citado 2019 mai 1]. Disponível em: https://pt.surveymonkey.com.

15. Minayo MCS. Pesquisa social: teoria, método e criatividade. $34^{\mathrm{a}}$ ed Petrópolis: Vozes; 2015.

16. David HMSL, Riera JRM, Mallebrera AH, Costa MFL. A enfermeira gestora de casos na Espanha: enfrentando o desafio da cronicidade por meio de uma prática integral. Cienc Saude Colet. 2020 jan;25(1):315-24. http:// dx.doi.org/10.1590/1413-81232020251.29272019. PMid:31859879.

17. Martins MM, Aued GK, Ribeiro OMPL, Santos MJ, Lacerda MR, Bernardino E. Gestão de alta para a continuidade do cuidado: experiência das Enfermeiras de Ligação de Portugal. Cogitare Enferm. 2018 set;23(3):e58449. http://dx.doi.org/10.5380/ce.v23i3.58449.

18. Dusek B, Pearce N, Harripaul A, Lloyd M. Care transitions: systematic review of best practices. J Nurs Care Qual. 2015;30(3):233-9. http:// dx.doi.org/10.1097/NCQ.0000000000000097. PMid:25470233.

19. Menezes TMO, Oliveira ALB, Santos LB, Freitas RA, Pedreira LC, Veras SMCB. Hospital transition care for the elderly: an integrative review. Rev Bras Enferm. 2019;72(suppl 2):294-301. http://dx.doi. org/10.1590/0034-7167-2018-0286. PMid:31826223.

20. Weber LAF, Lima MADS, Acosta AM. Quality of care transition and its association with hospital readmission. Aquichan. 2019;19(4): e1945 http://dx.doi.org/10.5294/aqui.2019.19.4.5.

21. Lima MADS, Magalhães AMM, Oelke ND, Marques GQ, Lorenzini E, Weber LAF, et al. Care transition strategies in Latin American countries: an integrative review. Rev Gauch Enferm. 2018 nov;39:e20180119. http://dx.doi.org/10.1590/1983-1447.2018.20180119.

22. Costa MFBNA, Ciosak SI, Andrade SR, Soares CF, Pérez EIB, Bernardino E. Continuidade do cuidado da alta hospitalar para a atenção primária à saúde: a prática espanhola. Texto Contexto Enferm. 2020 nov;29:e20180332. http://dx.doi.org/10.1590/1980-265x-tce-2018-0332.

23. Schaefer R, Zoboli ELCP, Vieira M. Sofrimento moral em enfermeiros: descrição do risco para profissionais. Texto Contexto Enferm. 2018;27(4):e4020017. http://dx.doi.org/10.1590/0104-07072018004020017.

24. Costa CS, Normann KAS, Tanaka AKSR, Cicolella DA. A influência da sobrecarga de trabalho do enfermeiro na qualidade da assistência. Rev Uningá. [internet] $2018 \mathrm{dez}$; [citado 2020 mai 11];55(4):110-20. Disponível em: http://revista.uninga.br/index.php/uninga/article/view/2403

25. Aires LCP, Santos EKA, Bruggemann OM, Backes MTS, Costa R Referência e contrarreferência do bebê egresso da unidade neonatal no sistema de saúde: percepção de profissionais de saúde da atenção primária. Esc Anna Nery. 2017;21(2):e20170028. http://dx.doi. org/10.5935/1414-8145.20170028.

26. Pereira KT, Sousa HA, Duarte SRMP. Efetividade dos resultados de serviço de contrarreferência em hospital quaternário do sul de Minas Gerais. Cienc Saude.2020 jan;10(1):17-22. http://dx.doi.org/10.21876/rcshci.v10i1.851.

27. Hermida PMV, Nascimento ERP, Echevarría-Guanilo ME, Andrade SR, Ortiga AMB. Counter-referral in emergency care units: discourse of the collective speech. Rev Bras Enferm. 2019 fev;72(suppl 1):143-50. http://dx.doi.org/10.1590/0034-7167-2018-0023. PMid:30942356.

28. Brown RA, Crookes PA. What are the 'necessary' skills for a newly graduating RN? Results of an Australian survey. BMC Nurs. 2016 abr;15(1):23. http://dx.doi.org/10.1186/s12912-016-0144-8. PMid:27051351.

29. Treviso P, Peres SC, Silva AD, Santos AA. Competências do enfermeiro na gestão do cuidado. Rev Adm Saúde. 2017 out;17(69). http://dx.doi. org/10.23973/ras.69.59

30. Bezerra Sobrinho A, Bernardo JMS, Alexandre AKS, Oliveira VL, LeiteSalgueiro CDB. Liderança do enfermeiro: reflexões sobre o papel do enfermeiro no contexto hospitalar. Rev Mult Psic. 2018;12(41):693-710. http://dx.doi.org/10.14295/idonline.v12i41.1238.

31. Santos TL, Laprano MGG, Conceição AP. Orientações de alta hospitalar para o desempenho do autocuidado após a cirurgia cardíaca: revisão integrativa. Rev Baiana Enferm. 2020 jul;34:e35284. http://dx.doi. org/10.18471/rbe.v34.35284.

aArtigo extraído de Trabalho de Conclusão de Curso intitulado Continuidade da assistência para Atenção Primária à Saúde: prática dos enfermeiros de um hospital universitário, de autoria de Lays Souza de Oliveira, orientado por Maria Fernanda Baeta Neves Alonso da Costa, defendido no ano de 2020 , Curso de Graduação em Enfermagem da Universidade Federal de Santa Catarina. 